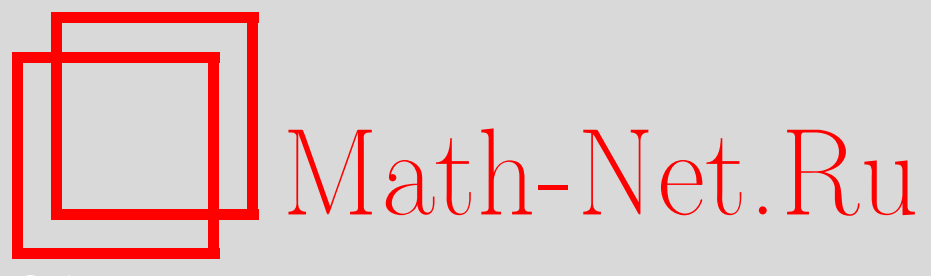

Р. К. Романовский, Л. В. Бельгарт, Об экспоненциальной дихотомии линейных разностных систем с почти периодической матрицей, Матем. заметки, 2008, том 84, выпуск 4, 638-640

DOI: https://doi.org/10.4213/mzm4147

Использование Общероссийского математического портала Math-Net.Ru подразумевает, что вы прочитали и согласны с пользовательским соглашением http://www.mathnet.ru/rus/agreement

Параметры загрузки:

IP : 54.162 .85 .209

26 апреля 2023 г., 11:23:46

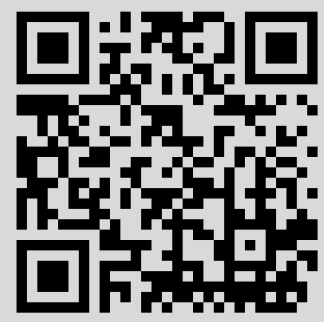




\section{Об экспоненциальной дихотомии линейных разностных систем с почти периодической матрицей}

\section{Р. К. Романовский, Л. В. Бельгарт}

Для автономной системы дифференциальных уравнений $\dot{x}=f(x), f(0)=0$, известен результат Барбашина [1], усиливающий теорему Ляпунова об асимптотической устойчивости: для асимптотической устойчивости положения равновесия $x=0$ достаточно существования положительно определенной функции $v(x)$ такой, что производная вдоль траекторий системы $\dot{v}(x) \leqslant 0$ и при этом поверхности уровня $v=$ const $>0$ не содержат целых траекторий. В работах [2], [3] показано, что этот результат распространяется на неавтономные системы $\dot{x}=f(x, t)$ при условии, что правая часть системы и функция Ляпунова $v(x, t)$ почти периодичны по $t$ (в случае линейных систем речь идет об экспоненциальной устойчивости). В [4] получен разностный аналог результата работы [2]. Рассматривается разностная система

$$
x_{n+1}=A_{n} x_{n}, \quad x_{n}: \mathbb{Z} \rightarrow \mathbb{C}^{N},
$$

где $A_{n}$ - почти периодическая матрица с отделенным от нуля определителем

$$
\left|\operatorname{det} A_{n}\right| \geqslant \text { const }>0 \text {. }
$$

Почти периодичность означает выполнение критерия компактности Бохнера [5]: семейство сдвигов $A_{n+k}, k \in \mathbb{Z},-$ предкомпакт в банаховом пространстве $\mathfrak{M}$ ограниченных функций $\mathbb{Z} \rightarrow \operatorname{Mat}(N, \mathbb{C})$ с равномерной нормой. Установлен признак экспоненциальной устойчивости в терминах эрмитовой формы

$$
v(x, n)=\left\langle G_{n} x, x\right\rangle
$$

с положительно определенной почти периодической матрицей $G_{n}$ с ослабленным условием на разностную производную формы (3) вдоль траекторий системы (1)

$$
\dot{v}(x, n)=\left\langle F_{n} x, x\right\rangle, \quad F_{n}=A_{n}^{*} G_{n+1} A_{n}-G_{n} ;
$$

для экспоненциальной устойчивости решения $x_{n}=0$ достаточно лишь, чтобы форма (4) была неположительна и отлична от тождественного нуля на каждом решении $x \neq 0$ системы (1). В основе обоснования лежат свойства некоторых функций на компакте $K=$ $H \times S$, где $H$ - оболочка почти периодической матрицы $A_{n}, S$ - единичная сфера в $\mathbb{C}^{N}$. В [6]-[12] результаты работ [2]-[4] распространены на другие классы почти периодических уравнений.

Данное сообщение является продолжением работы [4]. Исследуется в рамках прямого метода Ляпунова экспоненциальная дихотомия решений почти периодической разностной системы (1).

Обозначим через $U_{n}$ матрицу Коши системы (1): $x_{n}=U_{n} x_{0}$ для решений (1). В силу условия (2) матрица Коши определена при всех $n \in \mathbb{Z}$.

Будем, следуя [13], называть взаимным наклоном подпространств $E_{1}, E_{2}$ пространства $E=\mathbb{C}^{N}$ число

$$
\operatorname{Sn}\left(E_{1}, E_{2}\right)=\inf \left|z_{1}+z_{2}\right|, \quad z_{k} \in E_{k}, \quad\left|z_{k}\right|=1 .
$$

Здесь и далее $|\cdot|$ - эрмитова норма в $E$, так же обозначается согласованная с ней матричная норма. В частном случае, когда $E_{1}, E_{2}$ - вещественные прямые, $\operatorname{Sn}\left(E_{1}, E_{2}\right)=$ $2 \sin (\alpha / 2)$, где $\alpha$ - наименьший угол между прямыми.

(C) Р. К. РомановСКиЙ, Л. В. БЕЛьГАРт, 2008 
Будем говорить, что для системы (1) имеет место свойство экспоненииальной дихотомии (э-дихотомии), если фазовое пространство $E$ распадается в прямую сумму подпространств $E_{1}, E_{2}$ так, что

1) при некоторых $\mu_{1}, \nu_{1}, \mu_{2}, \nu_{2}>0$ выполняются оценки

$$
\begin{array}{llll}
x \in E_{1} & \Rightarrow & \left|U_{n} x\right| \leqslant \mu_{1} e^{-\nu_{1}(n-m)}\left|U_{m} x\right|, & n \geqslant m, \\
x \in E_{2} & \Rightarrow & \left|U_{n} x\right| \leqslant \mu_{2} e^{-\nu_{2}(m-n)}\left|U_{m} x\right|, \quad n \leqslant m,
\end{array}
$$

2) взаимный наклон движущихся подпространств $E_{k}(n)=U_{n} E_{k}$ отделен от нуля:

$$
\operatorname{Sn}\left(E_{1}(n), E_{2}(n)\right) \geqslant \text { const }>0 .
$$

В случае произвольной ограниченной $A_{n}$ для выполнения свойства э-дихотомии необходимо и достаточно существование эрмитовой индефинитной невырожденной ограниченной матрицы $G_{n}$ такой, что при некотором $m>0$ выполняется неравенство

$$
F_{n} \leqslant-m I \text {. }
$$

Обоснование проводится повторением с очевидными видоизменениями доказательства известного критерия э-дихотомии для систем $\dot{x}=A(t) x$ с непрерывной ограниченной матрицей $A(t)$ [14]; см. также [13; с. 280].

В классе почти периодических систем (1) имеет место усиление этого результата в сторону достаточности.

Обозначим через $\mathscr{J}$ класс матриц $G_{n}: \mathbb{Z} \rightarrow \operatorname{Mat}(N, \mathbb{C})$ со свойствами

1) $G_{n}^{*}=G_{n},\left|\operatorname{det} G_{n}\right| \geqslant$ const $>0$;

2) $G_{n}$ почтипериодична;

3) эрмитова форма (3) индефинитна.

Теорема 1. Если для системъ (1) с почти периодической матрицей $A_{n}$ существует матрица $G_{n} \in \mathscr{J}$ такая, что

$1^{\circ} \quad F_{n} \leqslant 0, n \in \mathbb{Z}$

$2^{\circ}$ форма (4) отлична от тождественного нуля на каждом решении $x_{n} \neq 0$ системы (1): $\dot{v}\left(x_{n}, n\right) \not \equiv 0$;

то система (1) э-дихотомична.

В основе обоснования лежат приемы, аналогичные развитым в [2]-[4].

ПримеР. Пусть в (1) $x_{n}: \mathbb{Z} \rightarrow \mathbb{R}^{2}$,

$$
A_{n}=\left[\begin{array}{cc}
\sqrt{1+a_{n}} & \sqrt{a_{n}^{-1}} \\
1 & \sqrt{1+a_{n}^{-1}}
\end{array}\right], \quad \varepsilon \leqslant a_{n}<1, \quad \varepsilon>0,
$$

$a_{n}-$ почти периодическая функция $\mathbb{Z} \rightarrow \mathbb{R}$. Покажем, что система $x_{n+1}=A_{n} x_{n}$ э-дихотомична.

Ввиду $a_{n} \geqslant \varepsilon$ матрица $A_{n}$ почти периодична, $\operatorname{det} A_{n}=\sqrt{a_{n}} \geqslant \sqrt{\varepsilon}$. Положим $G=$ $\operatorname{diag}(1 ;-1)$. Тогда

$$
F_{n}=A_{n}^{*} G A_{n}-G=\operatorname{diag}\left(a_{n}-1 ; 0\right) \leqslant 0 .
$$

Нетрудно убедиться: если $x_{n}=\left(\xi_{n}, \eta_{n}\right)$ - ненулевое решение системы $x_{n+1}=A_{n} x_{n}$, то $\xi_{n} \not \equiv 0$, поэтому

$$
\dot{v}\left(x_{n}, n\right)=x_{n}^{*} F_{n} x_{n}=\left(a_{n}-1\right) \xi_{n}^{2} \not \equiv 0 .
$$

Выполнены все условия теоремы 1 , тем самым утверждение доказано.

Отметим, что здесь не выполняется условие (5), гарантирующее выполнение свойства э-дихотомии при любой $a_{n}$ со свойством (6). 


\section{СПИСОК ЦИТИРОВАННОЙ ЛИТЕРАТУРЫ}

[1] Е. А. Барбашин, Функиия Ляпунова, Наука, М., 1970. [2] С. М. Добровольский, А. С. Котюргина, Р. К. Романовский, Матем. заметки, 52:6 (1992), 10-14. [3] С. М. Добровольский, Р. К. Романовский, Матем. заметки, 62:1 (1997), 151-153. [4] О.В.Кириченова, А. С. Котюргина, Р. К. Романовский, Сиб. матем. журн., 37:1 (1996), 170-174. [5] Б. М. Левитан, В.В. Жиков, Почти периодические функиии и дифференциальные уравнения, Изд-во МГУ, М., 1978. [6] Т. М. Стругова, Матем. заметки, 78:3 (2005), 472-475. [7] О. В. Кириченова, Сиб. матем. журн., 39:1 (1998), 45-48. [8] Н. В. Алексенко, Изв. вузов. Матем., 2000, № 2, 3-6. [9] Н. В. Алексенко, Р. К. Романовский, Дифферени. уравнения, 37:2 (2001), 147-153. [10] Р. К. Романовский, Г. А. Троценко, Сиб. матем. журн., 44:2 (2003), 444-453. [11] Г. А. Троценко, Изв. вузов. Матем., 2003, № 6, 77-81. [12] С. М. Добровольский, А. В. Рогозин, Сиб. матем. журн., 46:1 (2005), 98-105. [13] Ю. Л. Далецкий, М. Г. Крейн, Устойчивость решений дифференциальных уравнений в банаховом пространстве, Наука, М., 1970. [14] А. Д. Майзель, Тр. УрПИ. Сер. матем., 51 (1954), 20-50.

\section{Р. К. Романовский}

Поступило

Омский государственный технический университет

11.04 .2008

\section{Л. В. Бельгарт}

Омский государственный технический университет

E-mail: belgart@mail.rambler.ru 